\title{
Caregiver distress in parkinsonism
}

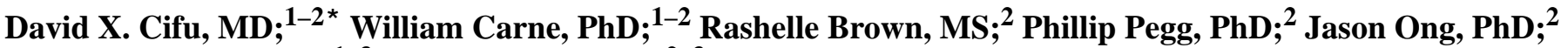 \\ Abu Qutubuddin, MD; ${ }^{1-3}$ Mark S. Baron, $\mathrm{MD}^{2-3}$ \\ ${ }^{1}$ Department of Physical Medicine and Rehabilitation, Virginia Commonwealth University, Richmond, VA; ${ }^{2}$ Southeast \\ Parkinson's Disease Research, Education, and Clinical Center, Hunter Holmes McGuire Department of Veterans \\ Affairs Medical Center, Richmond, VA; ${ }^{3}$ Department of Neurology, Virginia Commonwealth University, Richmond, VA
}

\begin{abstract}
This study examined the frequency and degree of caregiver burden in persons with parkinsonism, a group of disorders with four primary symptoms that include tremor, rigidity, postural instability, and bradykinesia. We assessed associations between perceived caregiver burden and physical, cognitive, and functional impairments using well-established tools for persons with parkinsonism. The 49 individuals with parkinsonism ranged in age from 61 to 87 (mean $=75$ ), while their caregivers $(N=49)$ ranged in age from 48 to 83 (mean $=$ $70)$. The caregivers were predominantly either wives $(82 \%)$ or daughters (6\%), with other family members, friends, and/or neighbors (12\%) making up the rest. The caregivers reported a relatively high ability for coping (mean scores $=4.6 / 6$ ). Caregiver burden was significantly negatively associated with activities of daily living and motoric difficulties as measured on the Unified Parkinson's Disease Rating Scale (UPDRS). Likewise, caregiver burden was negatively associated with caregiver self-reported sleep and coping ability. Results did not demonstrate an association on the UPDRS among mentation, behavior, and mood. We found a significant negative correlation for mentation between the Folstein Mini-Mental Status Examination and caregiver burden measures; however, we did not find this association with the Dementia Rating Scale-2. Patient's self-reported pain and caregiver burden were not associated.
\end{abstract}

Key words: aging parents, burnout, caregiver burden, caregiver distress, caregiver strain, disease progression, family burden, parkinsonism, Parkinson's disease, spousal support.

\section{INTRODUCTION}

The patient with a chronic debilitating disease frequently becomes increasingly reliant on a caregiver to assist with a multitude of daily tasks. Formal caregivers are paid helpers, while informal caregivers are unpaid friends or family members. According to Kasuya et al., "Informal or lay caregiving is the act of providing assistance to an individual with whom the caregiver has a personal relationship" [1, p. 119]. Caregivers are an intrinsic part of the patient's life and serve a useful role in the medical treatment process. As Ham has noted [2], the caregiver, as the single individual involved in the care of

\footnotetext{
Abbreviations: ADL = activities of daily living; CDS = Caregiver Distress Scale; DRS-2 = Dementia Rating Scale-2; H\&Y = Hoehn and Yahr; MMSE = Mini-Mental Status Examination; PADRECC = Parkinson's Disease Research, Education, and Clinical Center; PD = Parkinson's disease; PWP = persons with parkinsonism; $\mathrm{SD}=$ standard deviation; S\&E ADL = Schwab and England ADL; UPDRS = Unified Parkinson's Disease Rating Scale; VAMC = Department of Veterans Affairs medical center; VAS = visual analogue scale; ZBI = Zarit Burden Inventory.

* Address all correspondence to David X. Cifu, MD, Chief; Physical Medicine and Rehabilitation Services, Hunter Holmes McGuire VAMC, 1201 Broad Rock Road, Richmond, VA 23249; 804-675-5931; fax: 804-675-5939.

Email: dcifu@hsc.vcu.edu

DOI: 10.1682/JRRD.2005.08.0136
} 
the individual over the entire course of the disorder, may elect to be, or may essentially evolve into, the effective leader of the caring team. At home, informal caregivers help the patient with safety, medication compliance, activities of daily living (ADL), and social involvement. At the medical facility, informal caregivers can serve as accurate historians, first-hand observers of the patient's reactions to medications and treatments, and clarifiers of patient communication to medical personnel. Thus, they help the medical team obtain accurate and reliable information and they ensure the appropriateness of the home environment for the patient. For these reasons, supporting the functioning of caregivers is vital to the successful medical management and return to the community of individuals with chronic illness and disability.

The very nature of the caregiving role creates considerable stress or burden. Kasuya et al. described caregiver burden as "the strain or load borne by a person who cares for an elderly, chronically ill, or disabled family member or other person. . . . the point where the experience is no longer a viable or healthy option for either the caregiver or the person receiving care” [1, p. 119]. The authors further recognized a multitude of factors contributing to this burden to include physical, psychological, social, financial, and emotional stressors. As further noted, the shift in modern medical care to ambulatory/outpatient modes has increased the responsibilities of the patient's family caregivers [1]. Most families relinquish the caregiver function and accede to institutional care only in the terminal phases of an illness, if ever.

The issues of caregiver burden can affect the patient. At least two studies have suggested that the caregiver's sense of burden affects both the patient's functionality and adjustment to Parkinson's disease (PD) [3-4]. In this regard, McFall and Miller identified three factors that predict nursing home placement within a 2-year period, namely, advanced age, race (white), and instrumental ADL impairment [5]. The presence of these factors may suggest a link between individual caregiver "burnout" and placement of the individual with PD. Kasper et al. found that when a caregiver reached "absolute burnout," only 50 percent of patients were subsequently placed in an institution [6]. For the other half of the patients, other caregivers emerged and took over care. Even so, caregiver burden reduction (or "burnout" prevention) is well worth the time and effort, both in human and economic terms.
Data indicate that in most chronic illness cases in the United States, relatives rather than paid caregivers or institutional providers render care to patients [7]. The monetary implications of this finding are immense. The Parkinson's Action Network has calculated a cost for nursing facility care or hospitalization of $\$ 100,000$ annually per patient, therefore, emphasizing the caregiver's economic importance in maintaining the individual with PD in the home setting. Given the estimated prevalence of PD of between 500,000 and 1,500,000 in the United States and an annual incidence of 50,000 new cases, caregiving costs are clearly extensive [8-9]. While calculations of these costs are imprecise, the burden of nursing home stays, hospitalizations, and related medical care via public entitlements, such as Medicaid and Medicare, is considerable. Additionally, the hidden costs of caregivers' inability to work, disallowing their own income generation and paying of taxes, must be considered.

Although studies have demonstrated that caregiver burden exists in those caring for either predominantly physically ill patients (e.g., cancer, amyotrophic lateral sclerosis) or patients with cognitive impairments (e.g., traumatic brain injury, Alzheimer's disease), SandersDewey and colleagues noted that the study of caregiving factors in PD, which typically causes a combination of physical and cognitive deficits, has received little attention [10]. Because of the significant finding of increased caregiver burden in friends and family members of individuals with Alzheimer's disease [11-13], a similar increased burden would be likely in caring for individuals with PD. Supporting this concept, two recent studies demonstrated that caregiver burden in PD is associated with patient functional status, depressive symptoms, time since onset of PD, and other health-related issues [14-15]. Consequently, a sharper focus on the burden experienced by PD caregivers appears warranted.

The hallmarks of the most common form of parkinsonism, idiopathic PD, are bradykinesia, resting tremor, rigidity, festinating gait, and postural instability. As PD progresses, cognitive impairment is more likely, with dementia in the end stages often apparent. While the motor impairment emblematic of parkinsonism is the most notable symptom to the casual observer, some research suggests that cognitive, communication, and other less obvious problems associated with the disease progression create the most strain on family caregivers. In this vein, two Norwegian studies concluded that mental symptoms of PD patients were a major if not the strongest 
factor in creating caregiver distress [16-17]. Still other studies implicate motor impairment, nocturnal pain or cramping, and sleep fragmentation with caregiver distress [18]. While the exact determining factors in caregiver burden may be unclear, little doubt exists that the progressively debilitating nature of parkinsonism creates heavy and increasing demands on informal caregivers.

Caregiver burden is obviously an intrinsic yet understudied aspect of caring for persons with parkinsonism (PWP). Parkinsonism is the name given to a group of disorders (PD being one of them) with similar features with four primary symptoms, including tremor, rigidity, postural instability, and bradykinesia, resulting from the loss of dopamine-producing brain cells. Understanding the nature and mediators of caregiver burden would allow clinicians to more effectively treat caregivers and maintain them in the vital role of caring for PWP. In this study, we hypothesized that informal caregivers of PWP experience a significant amount of burden. Additionally, we hypothesized that disease progression, as measured by either physical or cognitive parameters, would be associated with greater caregiver burden. Finally, we examined the efficacy of a rapid screening tool to identify caregiver burden in those providing care to PD patients.

\section{METHODS}

The caregivers $(N=49)$ of all consecutive subjects referred with a movement disorder and seen for an initial or follow-up neuropsychological screening from July 2003 through May 2004 to the Parkinson's Disease Research, Education, and Clinical Center (PADRECC) at the Hunter Holmes McGuire Department of Veterans Affairs Medical Center (VAMC) in Richmond, Virginia, were included in this study. The PADRECC is a six-site program that began in 2000 and provides multidisciplinary care to all veterans with PD and related movement disorders [19]. We obtained approval for this study from the local VAMC institutional review board.

While all subjects were referred to this specialty clinic with a presumed diagnosis of primary PD, some were later found to not meet criteria; however, they were still included in this investigation because they met the criteria for parkinsonism. Because of the advanced age of this patient population, secondary comorbidities often existed and may have added to caregiver burden. During the initial evaluation of subjects, the identified primary informal caregiver was administered measures of caregiver burden as part of the standard clinical protocol. In addition to these measures, caregiver demographic information was obtained, along with self-reported number of sleep hours, health rating, and general coping level. All information was deidentified and password-protected when inputted into the database.

\section{Instruments}

\section{Caregiver Burden Instruments}

Zarit Burden Inventory. The Zarit Burden Inventory (ZBI) is a 22-item questionnaire with responses ranging from 1 to 4 and a total score range of 0 to 88 [20]. No cutoff scores have been established, but higher scores reflect higher caregiver burden. ${ }^{*}$ It is often used in the relevant literature as a measure of caregiver burden [21-22]. Each caregiver completed the ZBI in approximately 15 minutes.

Caregiver Distress Scale. The Caregiver Distress Scale (CDS) is a visual analogue scale (VAS) ranging from 1 to 10, with anchor points of No Stress (0), Moderate Stress (5), and Overwhelming Stress (10), that was developed in conjunction with the Richmond VAMC PADRECC program. The caregivers were asked to mark the scale at a point that best described their perceived degree of distress. The following supplementary information was also requested as part of the CDS. Two 6-point Likert scales were developed for the caregivers to rate their own health and general sense of coping. The caregivers were also asked to indicate how many hours they slept each night. Space for narrative comments regarding score selection factors and ways to lessen the burden was also provided. Words in the instruments were deleted if the investigators perceived them to be pejorative. Examples of such words are "burden” and "strain.” Each caregiver completed the CDS within 5 minutes.

Caregiver Survey. A caregiver survey is a brief program-specific internal PADRECC questionnaire that establishes the caregiver's relationship to the patient, current living situation, hours of caregiving provided weekly, need for the presence of a paid care provider, hours of sleep, health status, and coping status.

\footnotetext{
*Personal communication, email to William Carne from Steven Zarit referenced Norms ZBI. 2005 Mar 22.
} 


\section{Patient Instruments}

Mini-Mental Status Examination. The Folstein MiniMental Status Examination (MMSE) is probably the most widely used tool to screen for general cognitive impairment caused by dementia in the elderly. The MMSE is a 30-point structured clinician-rated interview scale incorporating pencil-and-paper tasks for assessing nine items: memory, orientation, attention, verbal fluency, nominal aphasia, receptive aphasia plus receptive apraxia, alexia, agraphia, and constructional apraxia [23].

Dementia Rating Scale-2. The Dementia Rating Scale2 (DRS-2) is a brief neuropsychological measure often used for individuals with known or suspected progressive neurocognitive impairment [24]. The DRS-2 has five subscales: attention (8 items), initiation/perseveration (11 items), construction (6 items), conceptualization (6 items), and memory (5 items). Total raw score equals 144.

Pain Visual Analogue Scale. The Pain VAS is a 10point Likert scale measurement collected from each subject at all clinic visits [25].

Unified Parkinson's Disease Rating Scale. The Unified PD Rating Scale (UPDRS) is accepted mostly for use in clinical research and drug trials that follow the longitudinal course of PD [26-28]. It is divided into four parts or scales: (1) mentation, behavior, and mood; (2) ADL; (3) motor; and (4) complications of therapy. In its entirety, UPDRS provides an overall assessment that quantifies all motor and behavioral aspects of PD. Each item is scored on scales ranging from 0 to 2 and from 0 to 4. A total of 16 points is possible on 4 items for mentation, behavior, and mood; 52 points for ADL on 13 items; 108 points for (bilateral) motor examination on 14 items; and 23 points for complications of therapy on 11 items. A score of 199 represents maximum (or total) disability, while 0 represents no disability.

Modified Hoehn and Yahr Staging Scale. As the fifth part of the original UPDRS, the modified Hoehn and Yahr (H\&Y) Staging Scale estimates disease staging [26,29]:

- 0 - No evidence of disease.

- 1.0-Unilateral disease only.

- 1.5-Unilateral disease plus axial involvement.

- 2.0-Bilateral mild disease without impaired balance.

- 2.5-Mild bilateral disease with recovery on pull test.

- 3.0 - Mild-to-moderate bilateral disease with some postural instability but physically independent.
- 4.0 — Severe disease, but still able to walk or stand unassisted.

- 5.0 - Wheelchair bound or bedridden unless aided. Modified Schwab and England Activities of Daily Living Scale. As the sixth part of the original UPDRS, the modified Schwab and England ADL (S\&E ADL) Scale is widely used for assessing disability in performing ADL. It is a percentage scale divided into deciles, with 100 percent representing completely normal function and 0 percent total helplessness [26].

\section{Patient and Caregiver Instruments}

We gathered demographics regarding caregiver race/ ethnicity, years of education, relationship to the PWP, and living situation. We gathered caregiver information on number of hours of care provided, help from others (paid or unpaid), and concern over leaving the patient alone. In addition, we collected patient information on driving ability and on whether or not the patient managed his or her own medications and finances.

\section{Statistical Analysis}

Data were analyzed with Statistical Package for Social Sciences (SPSS Inc, Chicago, Illinois), version 10.0, for Windows. Mean \pm standard deviation (SD) were calculated for each of the measures presented in this article and for continuous demographic variables (e.g., age). We used frequencies to examine categorical demographic variables (e.g., race/ethnicity). Bivariate correlations among the various measures were calculated with the degree of relationship between the indicators investigated through Pearson's correlation coefficients $(r)$. Tests of significance for all correlations were two-tailed, and given the presence of multiple correlations, we selected a more rigorous $p$-value of 0.01 to determine significance. We performed Spearman rank correlation coefficients $\left(r_{s}\right)$ to determine associations between patient characteristics (age, race/ethnicity, sex, marital status, level of education, pain, etc.), caregiver characteristics (age, level of education, hours of nightly sleep, perceived coping skills, perceived health status, etc.), patient disease status (i.e., UPDRS, S\&E ADL scale, and H\&Y scale), cognitive status (i.e., DRS-2 and MMSE), and the amount of caregiver distress reported by the veteran's primary caregiver (i.e., ZBI and CDS). We also calculated Spearman rank correlation coefficients $\left(r_{s}\right)$ to determine the relation between the ZBI and the CDS. 


\section{RESULTS}

\section{Patient Population Characteristics}

The 49 PWPs were all male and ranged from 61 to 87 years old $(75.0 \pm 5.4$, all values are mean \pm SD unless otherwise stated). Nearly 88 percent (43 patients) were white and 12 percent (6 patients) were African American (Table 1). The vast majority ( $n=39,80 \%)$ of the subjects had idiopathic PD, followed by a small number with essential tremor ( $n=4,8 \%)$, combined idiopathic PD with comorbid essential tremor ( $n=1,2 \%)$, Lewy body dementia $(n=2,4 \%)$, and other $(n=3,6 \%)$. Only 14 percent were still employed, with over 73 percent on medical or full retirement status. Approximately 84 percent were married, 10 percent widowed, and the remainder either separated $(2 \%)$ or divorced (2\%). One patient's marital status (2\%) was undetermined. The majority (88\%) lived with family, while only 2 percent lived alone, 6 percent lived with nonfamily members, and 4 percent failed to identify their living situation. Mean educational level was $11.6 \pm 3.5$ years (Table $\mathbf{1}$ ).

\section{Caregiver Population Characteristics}

Caregivers $(N=49)$ ranged in age from 48 to $83(70 \pm$ 9.9) and were significantly younger when compared with their partners with parkinsonism $(p=0.001)$ (Table 1). Caregivers were 59 percent white and 10 percent African American (over 30\% elected not to identify themselves by race/ethnicity) and ranged in educational level from 7 to 16 years $(11.7 \pm 2.5)$. No difference in education level was found between caregiver-subject pairs. By far, most of the caregivers were either wives (82\%) or daughters (6\%) (Table 1). Caregivers averaged $6.4 \pm 1.5$ hours of sleep nightly (range $=3-8$ ) (not shown in Table 1). When asked to scale their health in general on a 6-point Likert-type scale ( 1 = extremely poor and $6=$ extremely good), caregivers scored $4.6 \pm 0.8$ (range $=3-6$ ). When queried about their general sense of coping with the PD patient on a similar scale $(1=$ extremely poor and $6=$ extremely well $)$, they scored $4.6 \pm 0.9$ (range $=2-6$ ).

Caregiver burden, as measured by the CDS but not the ZBI, was significantly associated with performance of patients' ADL (UPDRS Part II and the S\&E ADL scale; $p<0.01)$. These results suggest that burden is associated with higher levels of patient difficulties with ADL. Motoric difficulties (UPDRS Part III) are also significantly associated with caregiver burden. Results did not confirm an association between mentation, behavior, and mood (UPDRS Part I) and caregiver burden (Table 2). In
Table 1.

Patient and caregiver demographics.

\begin{tabular}{|c|c|c|}
\hline Demographic & $\begin{array}{l}\text { Patients } \\
(N=49)\end{array}$ & $\begin{array}{c}\text { Caregivers } \\
(N=49)\end{array}$ \\
\hline \multicolumn{3}{|l|}{$\overline{\text { Age }}$} \\
\hline Mean \pm SD & $75^{*} \pm 5.4$ & $70^{*} \pm 9.9$ \\
\hline Range & $61-87$ & $48-83$ \\
\hline \multicolumn{3}{|l|}{ Race/Ethnicity (\%) } \\
\hline White & 88 & 59 \\
\hline African American & 12 & 10 \\
\hline Unidentified & 0 & 31 \\
\hline \multicolumn{3}{|l|}{$\operatorname{Sex}(\%)$} \\
\hline Male & 100 & 2 \\
\hline Female & 0 & 98 \\
\hline \multicolumn{3}{|l|}{ Marital Status (\%) } \\
\hline Married & 84 & - \\
\hline Separated & 2 & - \\
\hline Divorced & 2 & - \\
\hline Widowed & 10 & - \\
\hline Undetermined & 2 & - \\
\hline \multicolumn{3}{|c|}{ Relationship of Caregiver to Patient (\%) } \\
\hline Wife & 82 & - \\
\hline Daughter & 6 & - \\
\hline Son & 2 & - \\
\hline Daughter-in-Law & 2 & - \\
\hline Significant Other & 6 & - \\
\hline Friend & 2 & - \\
\hline \multicolumn{3}{|l|}{ Educational Level $^{\dagger}$ (yr) } \\
\hline Mean \pm SD & $11.6^{\dagger} \pm 3.5$ & $11.7^{\dagger} \pm 2.5$ \\
\hline Range & 2-19 & $7-16$ \\
\hline
\end{tabular}

addition, the total DRS-2 score did not correlate with the level of caregiver distress. Analysis also failed to demonstrate an association between the ZBI and CDS to any of the DRS-2 subscales (Table 2). However, the Folstein MMSE did correlate with the ZBI and the CDS $(p>0.01)$. The MMSE attention subscale negatively correlated with both caregiver burden measures ZBI and CDS ( -0.40 and -0.41 ), and the orientation subscale negatively correlated with only the CDS (-0.48) (Table 3).

Self-reported caregiver hours of sleep $(6.4 \pm 1.5, p \leq$ $0.01)$ and coping abilities (4.6/6 $\pm 0.93 ; p \leq 0.01)$ significantly negatively correlated with both measures of caregiver burden. Patient pain ratings, as well as self-reported caregiver health status, did not correlate with caregiver burden (Table 2). 
JRRD, Volume 43, Number 4, 2006

Table 2.

Pearson's correlation coefficients $(r)$ between clinical dimensions of parkinsonism and caregiver burden instruments.

\begin{tabular}{lcc}
\multicolumn{1}{c}{ Clinical Dimension } & Zarit Burden Inventory (r) & Caregiver Distress Scale (r) \\
\hline UPDRS & & 0.17 \\
Part I: Mentation, Behavior, and Mood & $0.55^{*}$ & 0.28 \\
Part II: ADL & $0.55^{*}$ & $0.46^{*}$ \\
Part III: Motor Examination & 0.04 & $0.54^{*}$ \\
Part IV: Complications of Therapy & -0.36 & -0.06 \\
Schwab \& England ADL Scale & 0.46 & $0.55^{*}$ \\
Hoehn and Yahr Staging Scale & $-0.45^{*}$ & $-0.51^{*}$ \\
Folstein Mini-Mental Status Examination & -0.26 & -0.20 \\
Dementia Rating Scale-2 & -0.01 & -0.05 \\
Pain Visual Analogue Scale & & $-0.562^{*}$ \\
Caregiver & $-0.682^{*}$ & -0.164 \\
$\quad$ Hours of Sleep & -0.227 & $-0.694^{*}$ \\
Health Status & $-0.561^{*}$ & \\
Perceived Coping Ability & & \\
\hline
\end{tabular}

${ }^{*} r$ is significant at 0.01 (two-tailed).

ADL = activities of daily living, UPDRS = Unified Parkinson’s Disease Rating Scale.

Table 3.

Pearson's correlation coefficients ( $r$ ) between Dementia Rating Scale-2 (DRS-2), Folstein Mini-Mental Status Examination (MMSE) subscales, and caregiver burden instruments.

\begin{tabular}{lccc}
\hline \multicolumn{1}{c}{ Cognitive Scale } & Mean \pm SD & Zarit Burden Inventory (r) & Caregiver Distress Scale (r) \\
\hline DRS-2 Total & $123.50 \pm 15.87$ & -0.26 & -0.20 \\
Attention & $34.20 \pm 3.63$ & -0.20 & -0.20 \\
Initiation/Perseveration & $29.10 \pm 7.30$ & -0.26 & -0.14 \\
Construction & $5.20 \pm 1.72$ & -0.34 & -0.29 \\
Conceptualization & $33.20 \pm 12.61$ & -0.32 & -0.01 \\
Memory & $21.60 \pm 13.36$ & -0.23 & 0.11 \\
Folstein MMSE & $26.70 \pm 3.98$ & $-0.45^{*}$ & $-0.51^{*}$ \\
Orientation & $9.40 \pm 1.33$ & -0.32 & $-0.48^{*}$ \\
Registration & $3.00 \pm 0.00$ & $\mathrm{NA}^{\dagger}$ & $\mathrm{NA}^{\dagger}$ \\
Attention & $5.60 \pm 2.72$ & $-0.40^{*}$ & $-0.41^{*}$ \\
Language & $8.60 \pm 0.65$ & -0.27 & -0.32 \\
${ }^{*} r$ is significant at 0.01 (two-tailed). & & \\
${ }^{\dagger}$ Could not be computed because all subjects had maximum score. & \\
MMSE $=$ Mini-Mental Status Examination, NA = not applicable, SD standard deviation. & \\
\hline \hline
\end{tabular}

The measures of caregiver burden used in this study, the ZBI and the visual analogue CDS, were highly and positively correlated $(r=0.696, p<0.01)$ with each other despite different approach measures (verbal/descriptive versus visual).

\section{DISCUSSION}

This study highlights the significant effect that the progressive physical and cognitive impairments common in parkinsonism have on persons providing care to PWPs. Despite the high prevalence of disability in PWP and the difficulties of caregiver burden reported in conditions with similar multifocal deficits such as Alzheimer's dementia and traumatic brain injury [11-13,30-32], this study represents one of the first to describe this burden for PWP. An improved understanding of the severity and correlates of caregiver burden in the parkinsonism patient population may allow clinicians to better prioritize treatment strategies for PWP and be more aware of caregivers' needs. 
Many clinicians may assume that a direct association exists between the physical impairments and concomitant caregiver burden of PWPs; however, this study suggests that this association is more complex. While an intuitively logical, significant relationship exists between measures of poorer motor and physical functioning (e.g., UPDRS Part III [motor examination], S\&E ADL scale, use of an assistive device) and caregiver burden, a significant association also exists between basic cognitive functioning, as measured by the MMSE and caregiver stress. These results support research by Carter et al. that suggests that cognitive deficits result in significant caregiver burden $[27,33]$.

Interestingly, more specific measures of dementia, and more specifically subcortical dementia (e.g., DRS-2 total score and five individual subscales), do not associate with levels of caregiver burden. Subcortical dementia commonly associated with parkinsonism is often characterized by memory loss with slowness in processing information, maintaining a consistent verbal response set, and responding intellectually. The type and degree of cognitive deficits demonstrated in this study (Table 3) do suggest difficulties with memory and initiation consistent with subcortical dementia as expected. However, the correlates of burden found in this study reflect more difficulties with attention and orientation measured by the MMSE. While these findings are not the typical clinical difficulties associated with PWP, this research suggests they are the deficits that correlate most closely with caregiver burden. Perhaps the inability of the PWP to easily interact with and remain focused on the needs or wishes of caregivers (related to orientation and attention difficulties) provides a greater degree of distress than the more typical subcortical deficits. Anecdotally, caregivers often remark on the communication difficulties created by poor attention and orientation. Because the lack of sensitivity of the more specific DRS-2 is counterintuitive, further investigation will be necessary for clinicians to understand the differences in the cognitive screening aspects of the MMSE and the DRS-2.

We studied two areas of caregiver self-report. The first area, the association of sleep and coping ability to caregiver burden, has important treatment implications. Sleep fragmentation in caregivers may affect both their physical and mental health maintenance. Clinicians need to be aware of and routinely inquire about caregivers' sleep and make appropriate referrals if necessary. Coping mechanisms similarly warrant detailed attention to help caregivers decrease burden. When clinicians work with caregivers, focusing on self-efficacy can be quite productive. The second area of caregiver self-report studied, health status, did not correlate with burden. This may be related to caregivers who tend to deemphasize their own well-being in lieu of their loved ones. Of interest, patient pain was not related to caregiver burden. Perhaps, pain may have been manifested with physical limitations rather than voiced complaints given the inherent cognitive and communication difficulties that are commonplace. Finally, this study did not identify age as an additional risk factor, although the small sample size of the younger dyads precluded valid statistical analysis. Further research should be done.

This study represents a preliminary descriptive study of the caregiver burden in consecutively enrolled PWP. Parkinsonism is an array of progressive neurologic conditions with significant variability in clinical manifestations. Because the severity of symptoms may fluctuate because of timing of medications and degree of physical activity, the level of caregiver burden may also fluctuate. Thus, caregiver burden may not be a static phenomenon in this patient population and a longer period of evaluation may be needed. The consecutive enrollment and prospective nature of this study were attempts to overcome subject selection bias; however, this may have been imperfect because more burdened individuals may have been more apt to seek treatment. Additionally, individuals with concurrent medical morbidities may have been more likely to be referred to this tertiary care center and again may have had higher associated caregiver burden. The complex nature of the Veterans Health Administration computerized clinical delivery system allowed an open access referral policy, which limited the gathering of all necessary clinical information (e.g., other relevant past medical or psychiatric history). Consequently, complete medication histories were not always available. While the UPDRS is the "gold standard" for the assessment of PD, it has limitations in interrater reliability. Additionally, significant variations in the UPDRS may have resulted from the "pulsatile" effects of some parkinsonism medications and specific timing of evaluations was not possible. In this study, UPDRS ratings were limited to two specialty-trained neurologists; however, some variability may have occurred. Finally, while the sample population was robust enough to allow for statistical power, the modest number of subjects and the lack of female cohorts may limit the generalizability of these results. 


\section{CONCLUSIONS}

Awareness of caregiver burden factors is important to the appropriate total management of the PWP. This study suggests that, while caregivers generally report good overall coping abilities, the increased motor impairment seen with PWP, the decreased ability to perform ADLs experienced in PWP, and the decreased sleep time experienced by some caregivers were all associated with greater caregiver burden. In contrast, patient mood factors and level of pain do not appear to be associated with the degree of caregiver burden. Patient mentation and its relationship to caregiver burden is less clear and requires further study.

\section{ACKNOWLEDGMENTS}

We would like to thank the Southeast PADRECC for assistance in data collection.

This material was unfunded at the time of manuscript preparation.

The content of this article is solely the responsibility of the authors and does not necessarily represent the official views of PADRECC.

The authors have declared that no competing interests exist.

\section{REFERENCES}

1. Kasuya RT, Polgar-Bailey P, Takeuchi R. Caregiver burden and burnout. A guide for primary care physicians. Postgrad Med. 2000;108(7):119-23. [PMID: 11126138]

2. Ham RJ. Evolving standards in patient and caregiver support. Alzheimer Dis Assoc Disord. 1999;13 Suppl 2:S27-35. [PMID: 10622676]

3. Edwards NE, Ruettiger KM. The influence of caregiver burden on patients' management of Parkinson's disease: implications for rehabilitation nursing. Rehabil Nurs. 2002; 27(5):182-86,198. [PMID: 12242868]

4. Lee PWH, Chang PCM, Fung ASM, Leung PWL. J Hong Kong. Issues in the care of elderly patients with Parkinson's disease: cognitive impairment, daily life adjustment, behavioural/emotional problems and caregiver burden. Coll Psychiatr. 1994;4:21-27.

5. McFall S, Miller BH. Caregiver burden and nursing home admission of frail elderly persons. J Gerontol. 1992;47(2): S73-79. [PMID: 1538078$]$
6. Kasper J, Steinbach U, Andrews J. Factors associated with ending caregiving among informal caregivers to the functionally and cognitively impaired elderly population. Baltimore (MD): Johns Hopkins University Press; 1990.

7. ASPE Research Notes [homepage on the Internet]. Washington (DC): Office of the Assistant Secretary for Planning and Evaluation, U.S. Department of Health and Human Services; c1993 Apr [cited 2005 Nov 15]. Available from: http://aspe.hhs.gov/daltcp/reports/rn05.htm

8. Lang AE, Lozano AM. Parkinson's disease. First of two parts. N Engl J Med. 1998;339(15):1044-53. [PMID: 9761807]

9. Lang AE, Lozano AM. Parkinson's disease. Second of two parts. N Engl J Med. 1998;339(16):1130-43. [PMID: 9770561]

10. Sanders-Dewey NE, Mullins LL, Chaney JM. Coping style, perceived uncertainty in illness, and distress in individuals with Parkinson's disease and their caregivers. Rehabil Psychol. 2001;46(4):363-81.

11. Clyburn LD, Stones MJ, Hadjistavropoulos T, Tuokko H. Predicting caregiver burden and depression in Alzheimer's disease. J Gerontol B Psychol Sci Soc Sci. 2000;55(1):S2-13. [PMID: 10728125]

12. Donaldson C, Burns A. Burden of Alzheimer's disease: helping the patient and caregiver. J Geriatr Psychiatry Neurol. 1999;12(1):21-28. [PMID: 10447151]

13. Newcomer R, Yordi C, DuNah R, Fox P, Wilkinson A. Effects of the Medicare Alzheimer's Disease Demonstration on caregiver burden and depression. Health Serv Res. 1999;34(3):669-89. [PMID: 10445897]

14. Caap-Ahlgren M, Dehlin O. Factors of importance to the caregiver burden experienced by family caregivers of Parkinson's disease patients. Aging Clin Exp Res. 2002;14(5): 371-77. [PMID: 12602571]

15. Martinez-Martin P, Benito-Leon J, Alonso F, Catalan MJ, Pondal M, Zamarbide I, Tobias A, De Pedro J. Quality of life of caregivers in Parkinson's disease. Qual Life Res. 2005;14(2):463-72. [PMID: 15892435]

16. Aarsland D, Larsen JP, Karlsen K, Lim NG, Tandberg E. Mental symptoms in Parkinson's disease are important contributors to caregiver distress. Int J Geriatr Psychiatry. 1999;14(10):866-74. [PMID: 10521886]

17. Thommessen B, Aarsland D, Braekhus A, Oksengaard AR, Engedal K, Laake K. The psychosocial burden on spouses of the elderly with stroke, dementia and Parkinson's disease. Int J Geriatr Psychiatry. 2002;17(1):78-84. [PMID: 11802235]

18. Happe S, Berger K. The association between caregiver burden and sleep disturbances in partners of patients with Parkinson’s disease. Age Aging. 2002;31(5):349-54. [PMID: 12242196] 
19. Carne W, Cifu DX, Marcinko P, Pickett T, Baron M, Qutubbudin A, Calabrese V, Roberge P, Holloway K, Mutchler B. Efficacy of a multidisciplinary treatment program on oneyear outcomes of individuals with Parkinson's disease. NeuroRehabilitation. 2005;20(3):161-67. [PMID: 16340097]

20. Zarit SH, Reever KE, Bach-Peterson J. Relatives of the impaired elderly: correlates of feelings of burden. Gerontologist. 1980;20(6):649-55. [PMID: 7203086]

21. Gill CE, Khurana RK. Caregiver burden in Shy-Drager syndrome. J Nerv Ment Dis. 2000;188(1):47-50. [PMID: 10665461]

22. Marsh L, Williams JR, Rocco M, Grill S, Munro C, Dawson TM. Psychiatric comorbidities in patients with Parkinson disease and psychosis. Neurology. 2004;63(2):293-300. [PMID: 15277623]

23. Folstein MF, Folstein SE, McHugh PR. "Mini-mental State.” A practical method for grading the cognitive state of patients for the clinician. J Psychiatr Res. 1975;12(3):189-98. [PMID: 1202204]

24. Green RC, Woodard JL, Green J. Validity of the Mattis Dementia Rating Scale for detection of cognitive impairment in the elderly. J Neuropsychiatry Clin Neurosci. 1995; 7(3):357-60. [PMID: 7580199]

25. Wallerstein SL. Scaling clinical pain and pain relief. In: Bromm B, editor. Pain measurement in man: neurophysiological correlates of pain. New York (NY): Elsevier; 1984.

26. Fahn S, Elton RL, Members of the UPDRS Development Committee. Unified Parkinson's Disease Rating Scale. In: Fahn S, Mardsen CD, Goldstein M, Calne DB, editors.
Recent developments in Parkinson's disease. New York (NY): Macmillan; 1987. p. 153-63.

27. Van Hilten JJ, Van der Zwan AD, Zwinderman AH, Roos RA. Rating impairment and disability in Parkinson's disease: evaluation of the Unified Parkinson's Disease Rating Scale. Mov Disord. 1994;9(1):84-88. [PMID: 8139609]

28. Richards M, Marder K, Cote L, Mayeux R. Interrater reliability of the Unified Parkinson's Disease Rating Scale motor examination. Mov Disord. 1994;9(1):89-91. [PMID: 8139610]

29. Hoehn MM. The natural history of Parkinson's disease in the pre-levodopa and post-levodopa eras. Neurol Clin. 1992;10(2):331-39. [PMID: 1584177]

30. Marsh NV, Kersel DA, Havill JH, Sleigh JW. Caregiver burden at 1 year following severe traumatic brain injury. Brain Inj. 1998;12(12):1045-59. [PMID: 9876864$]$

31. Sander AM, High WM Jr, Hannay HJ, Sherer M. Predictors of psychological health in caregivers of patients with closed head injury. Brain Inj. 1997;11(4):235-49.

[PMID: 9134198]

32. Smith AM, Schwirian PM. The relationship between caregiver burden and TBI survivors' cognition and functional ability after discharge. Rehabil Nurs. 1998;23(5):252-57. [PMID: 10067640]

33. Carter JH, Lyons KS, Stewart BJ, Archibald P. Do the clinical features of Parkinson's disease explain spouse caregiver strain? [abstract]. Neurology. 2002;58:A468.

Submitted for publication August 15, 2005. Accepted in revised form January 13, 2006. 